\title{
General calculation of $4 f-5 d$ transition rates for rare-earth ions using many-body perturbation theory
}

\author{
Chang-Kui Duan \\ Institute of Applied Physics and College of Electronic Engineering, \\ Chongqing University of Post and Telecommunications, Chongqing 400065, China. \\ Michael F. Reid \\ Department of Physics and Astronomy and MacDiarmid \\ Institute of Advanced Materials and Nanotechnology, \\ University of Canterbury, Christchurch, New Zealand
}

(Dated: November 9, 2018)

\begin{abstract}
The $4 f-5 d$ transition rates for rare-earth ions in crystals can be calculated with an effective transition operator acting between model $4 f^{N}$ and $4 f^{N-1} 5 d$ states calculated with effective Hamiltonian, such as semi-empirical crystal Hamiltonian. The difference of the effective transition operator from the original transition operator is the corrections due to mixing in transition initial and final states of excited configurations from both the center ion and the ligand ions. These corrections are calculated using many-body perturbation theory. For free ions, there are important one-body and two-body corrections. The one-body correction is proportional to the original electric dipole operator with magnitude of approximately $40 \%$ of the uncorrected electric dipole moment. Its effect is equivalent to scaling down the radial integral $\langle 5 d|r| 4 f\rangle$, to about $60 \%$ of the uncorrected HF value. The two-body correction has magnitude of approximately $25 \%$ relative to the uncorrected electric dipole moment. For ions in crystals, there is an additional one-body correction due to ligand polarization, whose magnitude is shown to be about $10 \%$ of the uncorrected electric dipole moment.
\end{abstract}




\section{INTRODUCTION}

In the recent years, optical spectroscopy of lanthanides ions in crystals involving $4 f-5 d$ transitions has been widely studied. These transitions are electric dipole parity allowed, and therefore suitable for efficient absorption of VUV radiation from the noble gas discharge in mercury-free lamps and plasma display panels. ${ }^{1.2 .3} \mathrm{~A}$ well-known application of the strong $4 f-5 d$ absorption of lanthanides is in blue lamp phosphors $\mathrm{BaMgAl}_{10} \mathrm{O}_{17}: \mathrm{Eu}^{2+}$. Potential applications for which VUV $5 d-4 f$ emission can be used are tunable VUV lasers and scintillator materials.

The transition rates are due to electric dipole moment contribution. The electric dipole matrix elements between $4 f$ and $5 d$ orbitals have been generally expected to dominate the electric dipole moment, and most of the calculations $\frac{4.6 .7}{40}$ are aware of consider only this zeroth-order contribution, which we shall refer to as the uncorrected electric dipole moment in the paper. Recently, various detailed comparisons between theory and experiment for free ions have shown that the zeroth-order calculations considerably over-estimated the $5 d \rightarrow 4 f$ spontaneous emission rates and configurational mixing needs to be included. ${ }^{8,9.10}$ Using the pseudorelativistic Hartree-Fock (HFR) approach of Cowan ${ }^{11}$, a configuration interaction (CI) calculation for free-ion $\mathrm{Ce}^{3+}$ includes both 12 odd-parity and 12 even-parity configurations gave radiative lifetimes agrees to experiment, $\frac{10}{10}$ while a single configuration calculation gives uncorrected radial integral $\langle 5 d|r| 4 f\rangle=0.0435 \mathrm{~nm}$, which underestimates the lifetime, i. e., overestimates the rates, by a factor of about 3. For rare-earth ions in crystals, the calculation of electric-dipole allowed $4 f-4 f$ two-photon absorption rates has shown that some contributions due to ligand excitations are very important. ${ }^{12}$ Since the ligand polarization contributions to two-photon transition moment are mainly due to mod-

ification of one-photon transition moment between $4 f^{N}$ and $4 f^{N-1} 4 d$ states, they are also potentially important to $4 f-5 d$ one-photon transitions.

Direct CI calculations similar to the one for $\mathrm{Ce}^{3+}$ are less feasible for rare-earth ions with near half-filled $4 f$ shell due to the large dimension of state spaces and become even more impractical when ligands orbitals are included, as in the cases of quantum chemical cluster calculations. A systematical method to take all those contributions into account in a reduced space is effective operator method. ${ }^{13.14 .15}$ In this method, the full Hilbert space timeindependent Hamiltonian $H$ is transformed into an effective Hamiltonian $H_{\text {eff }}$, which acts on 
the reduced space (referred to as model space) and gives upon diagonalization a set of exact eigenvalues and model space eigenvectors. For a time-independent operator $O$, an effective operator $O_{\text {eff }}$ may be introduced that gives the same matrix elements between the model space eigenvectors of $H_{\text {eff }}$ as those of the original operator $O$ between the corresponding true eigenvectors of $H$. Effective Hamiltonians $H_{\text {eff }}$ and transition operators $O_{\text {eff }}$ are often constructed by many-body perturbation theory (MBPT) with order by order approximation $\underline{15}$ and then represented by connected diagrams similar to Feynman diagrams. With those diagrams, perturbation calculations involving great number of many-body intermediate states transform into calculations involving summations over one- and two-particle states. Hence the calculations are usually greatly simplified and the results are much easy to interpret since we can find out transition mechanism from diagrams with important contributions. The calculations can also be easily used together will semi-empirical crystal Hamiltonian

for $4 f^{N}$ configuration $\frac{16}{\underline{16}}$ and its adaption to $4 f^{N-1} 5 d$ configuration,,$\frac{4}{,}$ and with more general ligand field approaches.

In this paper we do a general many-body perturbation calculation for the $4 f-5 d$ effective transition operator. The zeroth and first-order contributions to the effective electric dipole operator are presented in Sec. II in terms of diagrams and algebra expressions. In Sec. III we make further approximations to select out important contributions and discuss the possible consequences to $4 f-5 d$ one-photon and $4 f \rightarrow 4 f$ two-photon transitions.

\section{GENERAL MANY-BODY PERTURBATIVE EXPANSION FOR EFFECTIVE OPERATOR AND DIAGRAMMATIC REPRESENTATION}

We partition the Hamiltonian $H$ for the center-ion-ligand system as usual into three terms,

$H=H_{0}+V_{1}+V_{2}$

where $H_{0}$ is a zeroth order model Hamiltonian, $V_{1}$ and $V_{2}$ are the part of one-body spin-orbit and two-body Coulomb interactions, respectively, not included in $H_{0}$. Usually one choose $H_{0}$ to be spherical and spin independent, here we retain spin-independence but do not limit $H_{0}$ to be spherical, so that if necessary the strong crystal-field interactions for $5 d$ electron in crystals can be included in $H_{0}$, as in the calculation of $4 f \rightarrow 4 f$ two-photon absorption 
spectra in $\mathrm{Eu}^{2+}: \mathrm{CaF}_{2}{ }^{17}$ In general, the effect of crystal-field interactions to $f-d$ transitions is mainly through the effect to eigenstates of the center ion.

The eigenstates of $H_{0}$ are antisymmetric product of one-particle states from the center ion and ligand ions. The model space is chosen to contain the product of both $4 f^{N}$ and $4 f^{N-1} 5 d$ states with states of completely filled shells from the ligands.

The effective Hamiltonians can be calculated with a many-body perturbation expansion. 18 However, for $4 f^{N}$ energy levels of rare-earth ions, the phenomenological crystal-field Hamiltonian $\underline{\underline{19}}$ with adjustable parameters turns to produce better energy levels. The phenomenological crystal-field Hamiltonian has also been adapted to the calculation of $4 f^{N-1} 5 d$ energy levels. ${ }^{4}$ Since the eigenvectors of the phenomenological Hamiltonian can be used as the model space eigenvectors of the Hermitian effective Hamiltonian, we need only construct the Hermitian effective transition operator.

We consider transition due to electric dipole mechanism only. The transition operator is: $\mathbf{D}=-e \sum_{i=1}^{N} \overrightarrow{r_{i}}$.

In addition to the zeroth-order contribution included in most calculations, here we include also the first-order contribution. Using our latest results on effective operators and modified diagrams, $\stackrel{15,20}{ }$ we get the zeroth- and first-order diagrams for the effective transition operator as shown in Fig 1 .

Since the effective operator acts between eigenvectors of effective Hamiltonian, which are linear combinations of bases in model space, the incoming lines and outgoing lines in Fig [1 can only be $4 f$ orbitals and $5 d$ orbitals. The internal lines can be any orbitals of the center ion and ligands, as long as at any horizontal level at least one of them is a core or unoccupied orbital.

The evaluation rules have been summarized previously by Duan et al. ${ }^{20}$ For these diagrams in Fig. 11 the contribution from each diagram is proportional to the product of matrix elements of all vertexes divided by the net outflow energy (outflow energy taking away inflow energy) of the $V$ vertex in the diagram. The algebraic expressions are as follows:

$$
\begin{aligned}
& (a)=\sum_{m, n} a_{m}^{+} a_{n} \cdot \mathbf{D}_{m, n} \\
& (b)=\sum_{m, n} a_{m}^{+} a_{n} \cdot \sum_{i} \frac{\mathbf{D}_{m, i}\left(V_{1}\right)_{i, n}}{\epsilon_{n}-\epsilon_{i}}
\end{aligned}
$$




$$
\begin{aligned}
(c) & =\sum_{m, n} a_{m}^{+} a_{n} \cdot \sum_{i} \frac{\left(V_{1}\right)_{m, i} \mathbf{D}_{i, n}}{\epsilon_{m}-\epsilon_{i}} \\
(d) & =\sum_{m, n} a_{m}^{+} a_{n} \cdot \sum_{u, a}\left[\frac{\left(V_{2}\right)_{m u, n a} \mathbf{D}_{a, u}}{\epsilon_{n}+\epsilon_{a}-\epsilon_{m}-\epsilon_{u}}+\frac{\left(V_{2}\right)_{m a, n u} \mathbf{D}_{u, a}}{\epsilon_{m}+\epsilon_{a}-\epsilon_{n}-\epsilon_{u}}\right] \\
(e) & =\frac{1}{4} \sum_{m n, p q} a_{m}^{+} a_{n}^{+} a_{q} a_{p} \cdot \sum_{i} \frac{2\left(V_{2}\right)_{m n, p i} \mathbf{D}_{i, q}}{\epsilon_{m}+\epsilon_{n}-\epsilon_{p}-\epsilon_{i}} \\
(f) & =\frac{1}{4} \sum_{m n, p q} a_{m}^{+} a_{n}^{+} a_{q} a_{p} \cdot \sum_{i} \frac{2\left(V_{2}\right)_{m i, p q} \mathbf{D}_{n, i}}{\epsilon_{p}+\epsilon_{q}-\epsilon_{m}-\epsilon_{i}},
\end{aligned}
$$

where $m, n, p, q$ are valence orbitals, i.e., $f$ or $d$ orbitals, $i$ can be any core or unoccupied orbital of the center ion or ligand ions, $u$ can be a valence or unoccupied orbital of the center ion or an unoccupied orbital of the ligand ions, $a$ is a core orbital of the center ion or ligand ions. Note that the two-body matrix elements are antisymmetric, i.e.

$$
\left(V_{2}\right)_{a b, c d}=\left\langle a b\left|V_{2}\right| c d\right\rangle-\left\langle a b\left|V_{2}\right| d c\right\rangle .
$$

\section{FURTHER APPROXIMATIONS AND IMPORTANT CONTRIBUTIONS}

We make the following two approximations: (1) we follow the usual approximation by neglecting integrals contain overlap of $4 f$ orbitals of the center ion with ligand orbitals; (2) we neglect the extremely weak spin-orbit interactions between two orbitals $n l$ and $n^{\prime} l\left(n \neq n^{\prime}\right) ! \underline{11}$ With these approximations, the one-body contributions and two-body contributions can be greatly simplified and the strength can be calculated using structure data and HFR results for free ions.

\section{A. One-body contributions}

The zeroth-order term is the electric dipole between $4 f$ and $5 d$ orbitals, denoted by Fig प(a). The first-order diagrams Fig प(b,c) can be neglected with the second approximations above. This leaves the only one-body contributions from Fig प(d). Under the first approximation above, there are two kind of contributions from Figप(d): (1) the contribution with the two internal lines are orbitals of the center ion, which does not change much from free ion to ions in crystals; (2) the contribution with the two internal lines are orbitals of the ligands, which is usually denoted as "dynamical coupling" or "ligand polarization" 
contribution. The contribution due to ligand-center ion overlap are neglected under the first approximation since it contains $4 f$-ligand overlap.

The first contribution. For the case of free ions, since both the Coulomb interaction $V_{2}$ and the energy denominator in the expression for Fig 1(d) are spherically symmetric and spin-independent, the resulting interaction for Fig 1(d) can only be one-body spinindependent rank-1 spherical tensor, which is proportional to the electric dipole between $4 f$ and $5 d$ orbitals with opposite sign. This provides a solid ground for calculations adopting an effective radial matrix element $\langle 5 d|r| 4 f\rangle_{\text {eff }}$ scaled down from $\langle 5 d|r| 4 f\rangle$. This contribution is dominated by the terms with the pair of internal lines $(5 p, 5 d)$, which have small energy denominators and large matrix elements. After complex manipulation of coupling and recoupling coefficients, we get the effective operator in terms of radial integrals and (re)coupling coefficients as follows:

$$
\begin{aligned}
& \mathbf{D}_{\mathrm{eff}}[(d), 1]=-\delta \mathbf{D} \\
& \quad=\delta(-e\langle 5 d|r| 4 f\rangle)\left(a_{5 d}^{+} \tilde{a}_{4 f}\right)^{1}+\text { C. C. } \\
& \delta=\sum_{K, i_{1}, i_{2}} \frac{R^{K}\left(5 d i_{1} ; 4 f i_{2}\right)}{\left|\epsilon_{5 d}+\epsilon_{i_{1}}-\epsilon_{4 f}-\epsilon_{i_{2}}\right|} \frac{\left\langle i_{2}|r| i_{1}\right\rangle}{\langle 5 d|r| 4 f\rangle}\left[\delta_{K 1} \frac{\left\langle i_{2}|| C^{(1)} \| i_{1}\right\rangle^{2}}{3}\right. \\
& \left.+\left\{\begin{array}{ccc}
d & 1 & f \\
i_{1} & K & i_{2}
\end{array}\right\} \frac{\left\langle 5 d|| C^{(K)} \| i_{2}\right\rangle\left\langle i_{2}|| C^{(1)}|| i_{1}\right\rangle\left\langle i_{1}|| C^{(K)}|| 4 f\right\rangle}{\left\langle 5 d|| C^{(1)}|| 4 f\right\rangle}\right],
\end{aligned}
$$

where C. C. denotes the complex conjugative term, $\left(i_{1}, i_{2}\right)$ is a pair of single-electron radial wavefunction indices, with one being core orbitals and the other valence or unoccupied orbitals. The leading terms are

$$
\begin{aligned}
\delta \approx & \frac{R^{1}(5 d 5 p ; 4 f 5 d)\langle 5 d|r| 5 p\rangle}{\langle 5 d|r| 4 f\rangle}\left(\frac{2}{3\left(\epsilon_{4 f}-\epsilon_{5 p}\right)}+\frac{4}{15\left(2 \epsilon_{5 d}-\epsilon_{4 f}-\epsilon_{5 p}\right)}\right) \\
& +\frac{2 R^{2}(5 d 5 p ; 5 d 4 f)\langle 5 d|r| 5 p\rangle}{35\langle 5 d|r| 4 f\rangle\left(\epsilon_{4 f}-\epsilon_{5 p}\right)}+\frac{2 R^{4}(5 d 5 p ; 5 d 4 f)\langle 5 d|r| 5 p\rangle}{21\langle 5 d|r| 4 f\rangle\left(\epsilon_{4 f}-\epsilon_{5 p}\right)} \\
& -\frac{4 R^{3}(5 d 5 d ; 5 p 4 f)\langle 5 d|r| 5 p\rangle}{35\langle 5 d|r| 4 f\rangle\left(2 \epsilon_{5 d}-\epsilon_{5 p}-\epsilon_{4 f}\right)} .
\end{aligned}
$$

The values of $\delta$ calculated from the leading terms are 0.3571 and 0.3502 for $\mathrm{Ce}^{3+}$ and $\operatorname{Pr}^{3+}$ respectively. Note that $\delta$ or $\mathbf{D}_{\text {eff }}$ does not depend on the crystal environment.

For free ion $\mathrm{Ce}^{3+}$, since there is only one active electron in model space $4 f+5 d$, the whole effective operator is strictly a one-body operator, which is approximately proportionally 
to the original electric dipole $\mathbf{D}$. The lifetime values from recent measurement and CI calculation $\underline{\underline{10}}$ can be used to work out the effective $\langle 5 d|r| 4 f\rangle_{\text {eff }} \approx 0.025 \mathrm{~nm}$. Compared to the uncorrected HFR value $\langle 5 d|r| 4 f\rangle_{\text {eff }}=0.0435 \mathrm{~nm}, \frac{21}{\underline{2}}$ this gives an experimental $\delta$ value for $\mathrm{Ce}^{3+}$, which is $\left(1-\langle 5 d|r| 4 f\rangle_{\mathrm{eff}} /\langle 5 d|r| 4 f\rangle\right) \approx 0.43$.

The second contribution. Neglecting the overlap between orbitals of the center ion and those of the ligand ions, we can do a bipolar expansion to the coulomb interaction. We obtain the same result as has been given earlier by Reid and Richardson 22 in calculation of two-photon transitions:

$$
\begin{aligned}
\mathbf{D}_{\text {eff }}((d), 2)= & (-e) \sum_{k=2,4,6}\left\langle 5 d\left|r^{k-1}\right| 4 f\right\rangle[(k)(2 k-1)(2 k+1) / 3]^{1 / 2} \times \\
& \sum_{L} \bar{\alpha}_{L}\left(\epsilon_{5 d}-\epsilon_{4 f}\right) R_{L}^{-(k+1)}\left[C^{k}(L) C^{k-1(i)}\right]^{\mathbf{1}} \\
= & (-e\langle 5 d|r| 4 f\rangle) \sum_{k=2,4,6} \sqrt{2 k+1}\left(A^{k}\left(a_{5 d}^{+} \tilde{a}_{4 f}\right)^{k-1}\right)^{\mathbf{1}}+\text { C. C., } \\
A_{q}^{k}= & \sqrt{k} \frac{\langle 5 d|r(k-1)| 4 f\rangle\left\langle 5 d|| C^{k-1}|| 4 f\right\rangle}{\langle 5 d|r| 4 f\rangle} \sum_{L} \frac{\bar{\alpha}_{L}\left(\epsilon_{5 d}-\epsilon_{4 f}\right) C_{q}^{k}(L)}{R_{L}^{k+1}} .
\end{aligned}
$$

where $L$ labels ligands, $i$ labels the valence rare-earth electrons, and

$\bar{\alpha}_{L}(\omega)=\left.\frac{1}{3} \sum_{q} \sum_{c, u}\left\langle\left|\phi_{c}\right| r_{q} \mid \phi_{u}\right\rangle\right|^{2}\left(\frac{1}{\epsilon_{u}-\epsilon_{a}+\omega}+\frac{1}{\epsilon_{u}-\epsilon_{a}-\omega}\right)$

is the isotropic polarizability of ligand $L$. In the summation, $q$ is over the component of $r_{q}$, and $c$ and $u$ are over core and unoccupied orbitals of ligand $L$, respectively. Here we introduce dimensionless coefficients $A_{q}^{k}(k=2,4,6, q=-k,-k+1, \cdots, k)$ with associated operators properly normalized so that their values reflect the relative magnitudes of the contributions compared to uncorrected electric dipole moment.

For $\mathrm{Ce}^{3+}: \mathrm{CaF}_{2}$, we use the structure data for $\mathrm{CaF}_{2}$, setting $\bar{\alpha}_{L} \approx 10^{-3} \mathrm{~nm}^{3}$ for $F^{-}, \underline{22}$ and $\mathrm{Ce}^{3+}$ free-ion data ${ }^{21}$ to obtain the $A_{q}^{k}$ values. The nonzero coefficients are $A_{0}^{4}=\sqrt{14 / 5} A_{ \pm 4}^{4}=$ -0.08 and $A_{0}^{6}=-\sqrt{2 / 7} A_{ \pm 4}^{6}=0.0064$. The case for $4 f-5 d$ transitions of $\mathrm{Gd}^{3+}: \mathrm{LaF}_{3}$ is estimated to be similar. Therefore, corrections due to ligand polarization is not so important for $4 f-5 d$ transitions as the corrections due to the excited states of the center ion. The reason that the ligand polarization contributions are important for ${ }^{8} S_{7 / 2} \rightarrow{ }^{6} I_{J} 4 f \rightarrow 4 f$ two-photon absorption is due to superposition of contributions from different intermediate states: the transitions are between states with main components satisfying $\Delta L=6$, most part of the contribution due to the $4 f-5 d$ rank-one uncorrected electric dipole contributions 
cancels, but the rank-3 and rank-5 ligand polarization contributions do not cancel and become important.

\section{B. Two-body contributions}

The two diagrams Fig 1(e) and Fig 1(f) are complex conjugation of each other. To give a nonzero contribution, the single internal line can only be an orbital of the center ion due to the negligible overlap between $4 f$ and ligand orbitals. These two diagrams are likely to be dominated by the two terms with $5 p$ internal lines, which have energy denominators and matrix elements comparable to the important term in Fig 1(d). The two terms with $5 p$ internal lines, which are complex conjugation of each other, can be written as (with the complex conjugate term neglected)

$$
\begin{aligned}
& \mathbf{D}_{\text {eff }}[(e, f), 0]=\frac{1}{2} \sum_{m_{1}, m_{2}, m_{3}, m_{4}} a_{4 f m_{1}}^{+} a_{5 d m_{2}}^{+} a_{4 f m_{3}} a_{4 f m_{4}} \times \\
& \frac{\sum_{m_{5}}\left\langle 4 f m_{1} 5 p m_{5}\left|V_{2}\right| 4 f m_{3} 4 f_{m} 4\right\rangle\left\langle 5 d m_{2}|\mathbf{D}| 5 p m_{5}\right\rangle}{\epsilon_{4 f}-\epsilon_{5 p}}+\text { C.C. } \\
&=\left(\epsilon_{4 f}-\epsilon_{5 p}\right)^{-1} \sum_{m_{1}, m_{2}, m_{3}, m_{4}} a_{4 f m_{1}}^{+} a_{5 d m_{2}}^{+} a_{4 f m_{3}} a_{4 f m_{4}} \times \sum_{K} R^{K}(4 f 5 p ; 4 f 4 f)+\text { C.C. } \\
& \sum_{q m_{5}}(-1)^{q}\left\langle 4 f m_{1}\left|C_{q}^{K}\right| 4 f m_{3}\right\rangle\left\langle 5 d m_{2}|\mathbf{D}| 5 p m_{5}\right\rangle\left\langle 5 p m_{5}\left|C_{-q}^{K}\right| 4 f m_{4}\right\rangle+\text { C.C. } \\
&= d(K, Q)(-e\langle 5 d|r| 4 f\rangle) \frac{\left[\left(a_{4 f}^{+} a_{4 f}\right)^{K}\left(a_{5 d}^{+} \tilde{a_{4}}\right)^{Q}\right]^{1}}{2}+\text { C.C. }
\end{aligned}
$$

where $K=2,4, Q=K-1, K$, and $K+1$ and the dimensionless coefficients

$$
\begin{aligned}
& d(K, Q)=\frac{R^{K}(4 f 5 p ; 4 f 4 f)\langle 5 d|r| 5 p\rangle}{\left(\epsilon_{4 f}-\epsilon_{5 p}\right)\langle 5 d|r| 4 f\rangle} \times \\
& \left\langle 4 f \| C^{K}|| 4 f\right\rangle\left\langle 5 d \| C^{1}|| 5 p\right\rangle\left\langle 5 p \| C^{K}|| 4 f\right\rangle(-1)^{K+Q+1} 2 \sqrt{\frac{(2 Q+1)}{3(2 K+1)}}\left\{\begin{array}{ccc}
1 & 1 & 2 \\
3 & Q & K
\end{array}\right\} .
\end{aligned}
$$

The operators associated with $d(K, Q)$ are appropriated normalized so that the relative strengths can be reflected by these $d(K, Q)$ coefficients. The $d(K, Q)$ values for $\operatorname{Pr}^{3+}$ are given in Table【. These values show that the corrections due to two-body effective operators have a magnitude of $25 \%$ and can be important for rare-earth ions with two or more electrons in $4 f$ and $5 d$ shells. Since in general matrix elements of two-body operators are not proportional to those of one-body operators, the effect due to two-body effective operators cannot be 
fully accounted for by scaling the electric dipole operator.

All the different contributions to $4 f-5 d$ electric dipole transitions we considered and their relative magnitude are listed in Table [I] The uncorrected electric dipole term and one-body free-ion term are proportional to each other and form the main contribution to $4 f-5 d$ transitions, which are equivalent to scaling the $4 f-5 d$ electric dipole radial integral with a factor $60 \%(=1-40 \%)$. We call this corrected one-body free-ion term. The next important contribution is the two-body free-ion term, which has a relative magnitude of about $25 \%$ of the uncorrected electric dipole term and $40 \%(\approx 25 \% / 60 \%)$ of the corrected one-body free-ion term. This term has the same rank as corrected one-body free-ion term (rank-1), but is two-body and cannot be fully accounted for by scaling the $4 f-5 d$ electric dipole radial integral. The ligand-polarization term is about $10 \%$ or the uncorrected electric dipole and $15 \%$ of corrected one-body free-ion term. This term is less important than two-

body free-ion term for $4 f-5 d$ transitions but the high rank (rank-3 and rank-5) part can be important for $4 f-4 f$ two-photon transitions with $\Delta L>2$.

\section{SUMMARY}

In summary, we have presented a first-order many-body perturbation calculation of the effective operator for $4 f-5 d$ transitions to account for the main corrections to the transition rates due to mixing of other configurations of the center ion and ligand orbitals in $4 f^{N}$ and $4 f^{N-1} 5 d$ states. Further approximations are made to select out possible important corrections to the usual zeroth-order $4 f-5 d$ electric dipole operator. First-order one-body contributions due to excited states of the center ion is shown equivalent to scale down the electric dipole radial integral $\langle 5 d|r| 4 f\rangle$, which, for $\mathrm{Ce}^{3+}$ and $\operatorname{Pr}^{3+}$, has almost reduced the effective value to about $60 \%$ of the uncorrected values. For rare-earth ions with two or more electrons in $4 f$ and $5 d$ open shells, first-order two-body contributions also have important contributions, which cannot be accounted for by scaling the electric dipole radial integral $\langle 5 d|r| 4 f\rangle$. The magnitudes have been calculated to be about $25 \%$ relative to the uncorrected electric dipole moment for $\operatorname{Pr}^{3+}$. Contributions due to ligand polarization are rederived, which are the same as given earlier by Reid and Richardson. ${ }^{22}$ The magnitude is about $10 \%$ relative to the uncorrected electric dipole moment for $\mathrm{Ce}^{3+}: \mathrm{CaF}_{2}$. The contribution due to ligand-center ion overlap is negligible, since it contains the overlap between $4 f$ and ligand 
orbitals.

\section{Acknowledgment}

C. K. D. acknowledges support of this work by the National Natural Science Foundation of China, Grant No. 10274079 and 10404040. 
1 B. Blasse and B. Grabmaier, Luminescent Materials (Springer Verlag, 1994), p. 130.

2 C. R. Ronda, J. Alloys Compd. 225, 534 (1995).

3 R. T. Wegh, H. Donker, K. D. Oskam, and A. Meijerink, Science 283, 593 (1999).

4 M. F. Reid, L. van Pieterson, R. T. Wegh, and A. Meijerink, Phys. Rev. B 62, 14744 (2000).

5 C. K. Duan and M. F. Reid, J. Solid State Chem. 171, 299 (2003).

6 A. Collombet, Y. guyot, M. F. Joubert, M. Laroche, and E. Descroix, Phys. Rev. B 68, 035115 (2003).

7 V. A. Dzuba, U. I. Safronova, and W. R. Johnson, Phys. Rev. A 68, 032503 (2003).

8 Z. S. Li, Z. G. Zhang, V. Lokhnygin, S. Svanberg, T. Bastin, E. Biemont, H. Garnir, P. Palmeri, and P. Quinet, J. Phys. B 34, 1349 (2001).

9 E. Biemont, H. P. Garnir, T. Bastin, P. Palmeri, P. Quinet, Z. Li, Z. Zhang, V. Loknygin, and S. Svanberg, Mon. Not. R. Astron. Soc. 321, 481 (2001).

10 Z. G. Zhang, S. Svanberg, P. Quinet, P. Palmeri, and E. Biemont, Phys. Rev. Lett. 87, 273001 (2001).

11 R. D. Cowan, The theory of atomic structure and spectra (University of California Press, Berkeley, Los Angeles and London, 1981).

12 G. W. Burdick, M. F. Reid, C. K. Duan, J. Chong, and A. Burdick (2003), presented on International Conference on Dynamical Processes of Excited States in Solids, Christchurch, New Zealand.

13 I. Lindgren, Rep. Prog. Phys. 47, 345 (1984).

14 V. Hurtubise, J. Chem. Phys. 99, 265 (1993).

15 C. K. Duan and M. F. Reid, J. Chem. Phys 115, 8279 (2001).

16 W. T. Carnall, G. L. Goodman, K. Rajnak, and R. S. Rana, J. Chem. Phys. 90, 3443 (1989).

17 C.-K. Duan, M. F. Reid, and G. W. Burdick, Phys. Rev. B 66, 155108 (2002).

18 B. H. Brandow, Rev. Mod. Phys. 39, 771 (1967).

19 D. J. Newman and B. K. C. Ng, Crystal Field Handbook (Cambridge University Press, 2000).

20 C. K. Duan, G. Ruan, and M. F. Reid, J. Chem. Phys. 121, 5071 (2004).

21 M. F. Reid, L. van Pieterson, and A. Meijerink, J. Alloys Compds. 344, 240 (2002).

22 M. F. Reid and F. S. Richardson, Phys. Rev. B 29, 2830 (1984). 


\section{Figures}

FIG. 1: Zeroth and First order diagrams for the effective electric dipole transition operator, where the free lines are $4 f$ and $5 d$ orbitals, the internal lines in diagrams (b), (c), (e) and (d) are core or unoccupied orbitals, one of the two internal lines in (d) is core orbital and the other can be unoccupied or valence orbitals. The rules to evaluate these diagrams can be found in Ref.20. 


\section{Tables}

TABLE I: The values of dimensionless coefficients for $\mathrm{Pr}^{3+}$ two-body transition effective operators, where $d_{\text {tot }}=\left(\sum d(K, Q)^{2}\right)^{1 / 2}$ denote the total magnitude of two-body interactions.

TABLE II: List of different contributions to $4 f-5 d$ transitions and their relative magnitude 
Figure 1. Duan and Reid, Journal of Chemical Physics

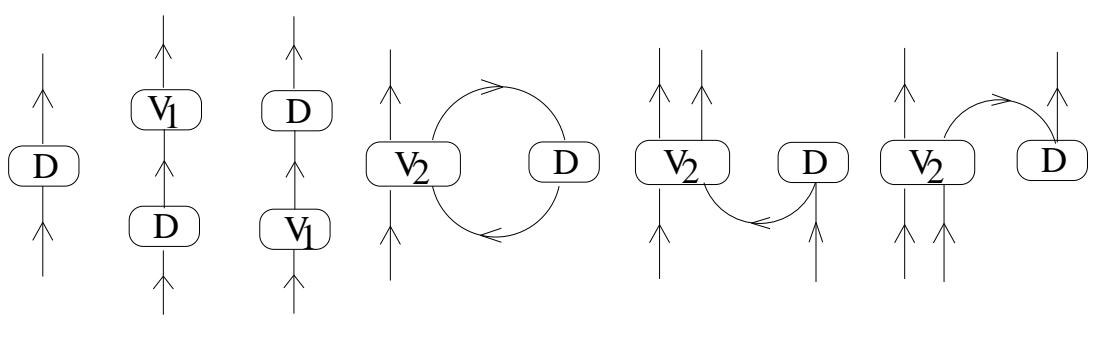
(a)
(b)
(c)
(d)
(e)
(f) 
Table [1. Duan and Reid, Journal of Chemical Physics

\begin{tabular}{llllllr}
\hline \hline$d(21)$ & $d(22)$ & $d(23)$ & $d(43)$ & $d(44)$ & $d(45)$ & $d_{\text {tot }}$ \\
-0.147 & -0.155 & -0.113 & -0.015 & -0.035 & -0.063 & 0.253 \\
\hline \hline
\end{tabular}


Table II. Duan and Reid, Journal of Chemical Physics

\begin{tabular}{cccc}
\hline \hline uncorrected & one-body free-ion & two-body free-ion & ligand-polarization \\
1 & $(-) 40 \%$ & $25 \%$ & $10 \%$ \\
\hline \hline
\end{tabular}

